\title{
Statyba
}

\section{EINE ANALYSE VON ZYKLISCHEN SYSTEMEN UNTER UNBESTIMMTHEITSBEDINGUNGEN}

\section{O. Kapliñski}

To cite this article: O. Kapliñski (1995) EINE ANALYSE VON ZYKLISCHEN

SYSTEMEN UNTER UNBESTIMMTHEITSBEDINGUNGEN, Statyba, 1:1, 93-101, DOI:

10.1080/13921525.1995.10531505

To link to this article: https://doi.org/10.1080/13921525.1995.10531505

曲 Published online: 26 Jul 2012.

Submit your article to this journal

山 Article views: 50 


\section{EINE ANALYSE VON ZYKLISCHEN SYSTEMEN UNTER UNBESTIMMTHEITSBEDINGUNGEN}

\section{O. Kapliñski}

\section{Zyklische Systeme im Bauwesen}

Eine Reihe von Bauprozessen sind durch sich widerholende technologische Tätigkeiten gekennzeichnet. Sie sind abhängig von der Realisierung vorgelagerter oder nachfolgender Stufen. Als Beispiel für zyklische Prozesse können genannt werden: Transportprozesse, das Zusammenwirken von Lademaschinen mit Transportmitteln, das Auswechseln von Formen in der Betonfertigteilproduktion u.s.w.. Als Attributmerkmal für harmonisierte Bauprozesse läßt sich folgende Bedingung schreiben:

$$
\mu_{1} \approx \mu_{2}, \ldots, \mu_{i}, \ldots, \approx \mu_{N}
$$

mit $\mu_{i}$ - Intensităt des Prozesse, $i=1,2, \ldots, N$,

$N$ - Zahl der Prozesse.

Für zyklische Prozesse (oder Systeme) besteht das Verhältnis

$$
\mu_{N+1}=\mu_{i} .
$$

Im Spezialfall $N=2$ ist $\quad m=\frac{\mu_{1}}{\mu_{2}} \quad\left(\mu_{1}<\mu_{2}\right)$

die Zahl der Parallelstellen und Brigaden oder

$$
m^{*}=\frac{\mu_{1}+\mu_{2}}{\mu_{1}}
$$

die Zahl der zirkulierenden Transporteinheiten; $i=1$ Fahrt, $i=2$ Beladung.

In einer deterministischen Fassung machen die o.g. Ausdrücke keine größeren Berechnungschwierigkeiten. In der stochastischen Fassung jedoch, müssen sie den erforderlichen Bedingungen entsprechen. Es besteht dann die Möglichkeit der Abbildung zyklischer Prozesse durch Bedienungssysteme (Warteschlagentheorie) oder mit Hilfe eines beschränkten Fordenungsstromes. 


\section{Unbestimmtheit}

Die sich ståndig ändernde Spezifik der Bauprozesse, die Schwierigkeiten bei der Beschaffung eines wahrhaftigen statistischen Materials bedingen, daß die Bauproduktion tatsächlich unter den Bedingungen einer Bestimmtheit realisiert wird (vgl. [1], [5], [7]. [8], und [12]). Sie sind charakterisiert durch das Auftreten mindestens eines Parameters, der nur durch die Form einer Wertmenge, die er annehmen kann, bestimmt wird (vgl. [6], [10], [11] und [13]).

\section{Analyse des zyklischen Systems}

Zum Gegenstand der Analyse werden die zyklischen Systeme, die die Wirkung der Bauprozesse abbilden. Die Analyse hat zum Ziel, eine Harmonisierung der Wirkungsweise dieser Systeme und auf dieser Grundlage die Bestimmung folgender Elemente: Maschinenkombination, die Zusammensetzung der Arbeitsbrigaden, die Vorbereitung der Arbeitsfront usw. Da die Analyse auf die Anwendung von strategischen Zwei-Personen-Spielen gegen die Natur basiert, sieht die Verfahrensprozedur wie folgt aus:

- es werden nacheinander zwei benachbarte Prozesse analysiert,

- es erfolgt eine Beschränkung auf zwei Hauptprozessen durch Reduzierung, Zurückführung komplizierter Systeme auf einfache mit zwei Zyklen (Prozessen).

Ein solches Verfahren wird oft in der Praxis benutzt, und es vollzieht sich auf der Grundlage der homomorphischen Umwandlungen, bei der Beibehaltung der Regeln der Dekomposition und Synthese des Systems (vgl. Abb. 1 - 4). Beispiele für ein solches Verfaren können in den Abbildungen 1, 2 und 3 verfolgt werden. Die dort dargestellten Beispiele der zyklischen Systeme können auf das Prinzip von Abbildung 4 zurückgeführt werden. Die Abb. 1 zeigt das Zusammenwirken eines Baggers mit Kippfaharzeugen. Dieses System kann auch auf ein ăhnliches reduziert werden, wie ein Schubfahrzeug und Schrapper (Abb. 2).

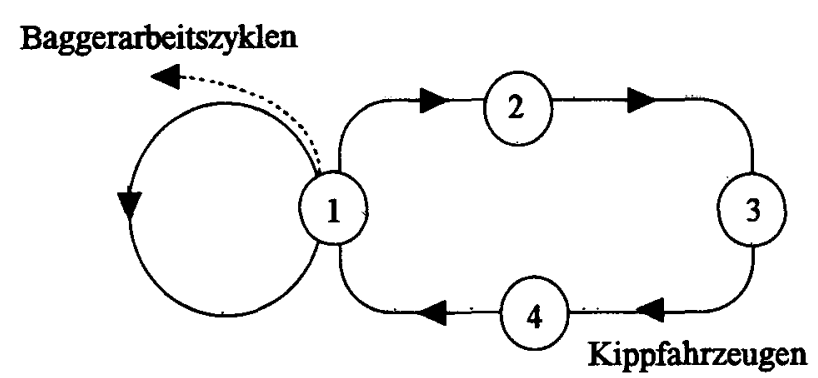

Abb. 1 


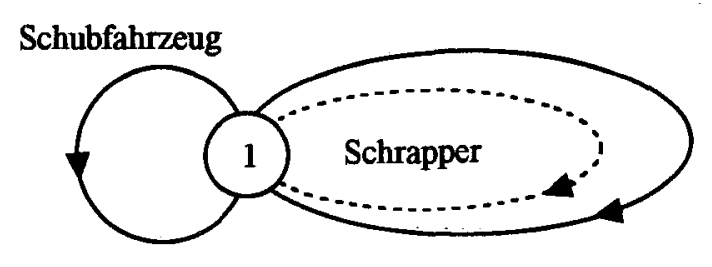

Abb. 2

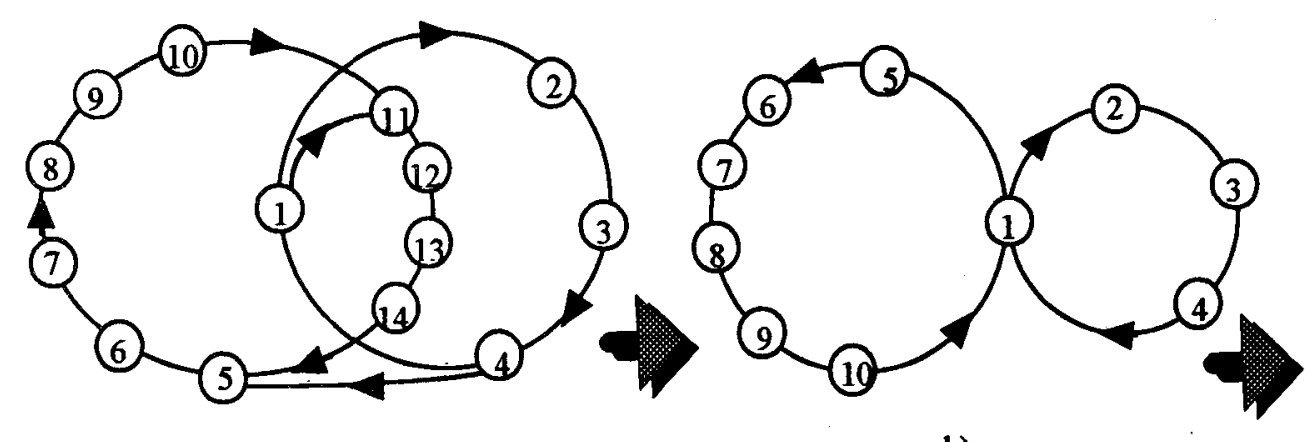

a)

b)

Abb. 3

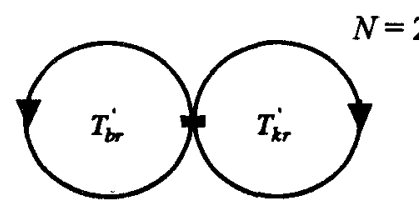

a)

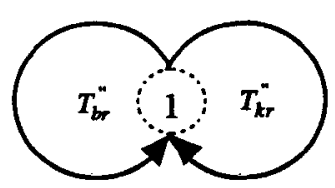

b) $T^{\prime \prime}=T^{\prime}-t_{1}$

Abb. 4

Die Abbildung 3a stellt ein kompliziertes System dar. Dabei handelt es sich um den Montageprozeß von Tunnelformvorrichtungen beim monolythischen Wohnungsbau. Dabei treten folgende Tătigkeiten auf: 1 - Montage, 2 - Leerlauf, 3- Aufhängung, 4 - Transport, 5 - Schießen, 6 - Justierung, 7 - Wandklammern, 8 - Eckklammern, 9 - Anker, 10 - Keilen und Laschen. Nach dem ersten Reduktionsschritt erhalten wir ein System wie in Abb. 3b und nach dem zweiten wie in Abb.4. Dabei sind: $1-4$ - Tătigkeiten des Kranes, 5 - 14 - Tătigkeiten der Baubrigade (Team).

Es besteht die Möglichkeit einer Analyse in Anlehnung an die so gen. erschütterte Gleichgewichtserscheinung bei unendlichem Strategie-Repertoire der beiden Spieler. Mit dieser 
Methode besteht die Möglikeit einer Korrektur der Nennbetriebsleistung der beiden Prozesse (Zyklen). Es handelt sich hier um die Erhaltung der ursprünglich angenommenen Leistung. Die Modellierung erfolgt dabei mit Hilfe der Verlustfunktion. Die erschütterte Gleichgewichtserscheinung wird beim Suchen des oberen Spielwertes erreicht. Dieses Verfahren wurde in [2] und [3] dargestellt. Eine für das Bauwesen geeignete Verlustfunktion $S$, die das Maß des Harmonisierungsmangels beschreibt, kann wie folgt ausgedrückt werden:

$$
S=\left|W_{1}-W_{2}\right|
$$

Bei Berücksichtigung ökonomischer Aspekte findet die Formel folgende Verănderung

$$
S= \begin{cases}C_{1}\left(W_{1}-W_{2}\right), & \text { fur } W_{1}-W_{2}>0 \\ C_{2}\left(W_{1}-W_{2}\right), & \text { fur } W_{2}-W_{1}>0\end{cases}
$$

mit $C_{1}, C_{2}$ - Kosten für Haupt- und Hilfsmaschinen. Vorschläge dieser Funktion und der verkürzte Optimierungsproze $B$ nach dem Wald-Kriterium sind in Tabelle 1 dargestellt.

\section{Beispiele der Analyse}

Die Analyse wurde auf der Grundlage von Abb. 3 geführt, wobei das System, wie in Abb. 4 dargestellt, reduziert wurde. Für die Analyse bestehen zwei Möglichkeiten:

- Fall 1: wenn uns die ganze Zeit des Prozesses (Zyklus) interessiert; Abb. 4a,

- Fall 2: wenn die Zeit einer engen Zusammenarbeit, z.B. die Tătigkeiten, die als "1" in der Abb. $4 \mathrm{~b}$ bezeichnet wurden, ausgeschlossen ist.

Die Aufgabe besteht in der Auswahl einer solchen Zahl $l_{s}$ (z.B. Mitglieder eines Montage-Teams), daB im Zeitraum des zweiten Zyklus (z.B. der Kranarbeit) einen bestimmten Umflang von Tătigkeiten, die geplant worden sind, unter Bedingung eines minimalen Stillstandes, leisten könnte.

Es werden folgende Bezeichnungen eingeführt

$T_{k r}\left(l_{s}\right)$ - die Zykluszeit des Kranes bei der Montage der Formvorrichtung,

$T_{b r}\left(l_{s}\right)$ - die Zeit, in der das Team mit einer Mitglieder-Zahl $l_{s}$ einen bestimmten Umflag von Tätigkeiten ausübt.

Dan erhalten die Funktionen $S_{a}$ (vgl. Tabelle 1) folgende Form

$$
S=\left|T_{k r}\left(l_{s}\right) n_{p}-T_{b r}\left(l_{s}\right) n_{s}\right|, \quad[\mathrm{h}]
$$




\begin{tabular}{|c|c|c|}
\hline $\begin{array}{c}\text { Funktion } \\
\text { der Verluste } S\end{array}$ & $F=\max _{n_{s}, n_{p}} S$ & $l_{s}$ für $F=\min$ \\
\hline$S_{a}=\left|Q_{t} l_{s} n_{s}-M n_{p}\right|$ & 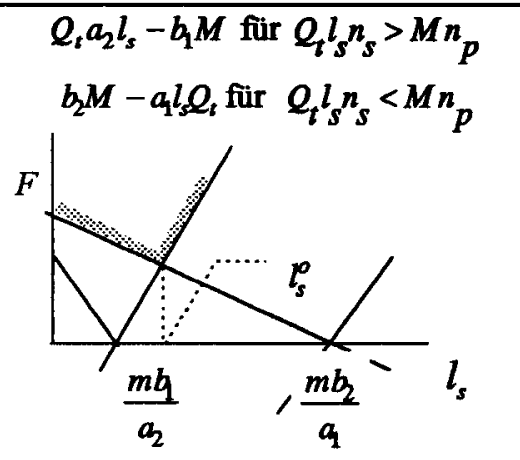 & $\begin{array}{c}\frac{m\left(b_{1}+b_{2}\right)}{a_{1}+a_{2}} \\
\text { wobei: } \\
m=\frac{M}{Q_{t}} \\
a_{1} \neq 0\end{array}$ \\
\hline$S_{b}=\left(Q_{t} l_{s} n_{s}-M n_{p}\right)^{2}$ & $\begin{array}{l}\left(Q_{t} a_{2} l_{s}-b_{1} M\right)^{2} \text { für } Q_{t} l_{s} n_{s}>M n_{p} \\
\left(Q_{t} a_{1} l_{s}-b_{2} M\right)^{2} \text { für } Q_{t} l_{s} n_{s}<M n_{p} \\
\underbrace{F}_{\frac{m b}{a_{2}}} \frac{m b_{s}}{a_{1}} l_{s}\end{array}$ & $\frac{m\left(a_{2} b_{1}-a_{1} b_{2}+a_{2} b_{2}-a_{1} b_{1}\right)}{a_{2}^{2}-a_{1}^{2}}$ \\
\hline$S_{c}=\left|\frac{n_{s}}{n_{p}}-\frac{m}{l_{s}}\right|$ & $\overbrace{\frac{m b}{a_{2}}} \frac{m b_{2}}{a_{1}}$ & $\begin{array}{l}\frac{2 m}{\frac{a_{2}}{b_{1}}+\frac{a_{1}}{b_{2}}} \\
b_{1}>0 \\
b_{2}>>0\end{array}$ \\
\hline \multicolumn{3}{|c|}{$\begin{array}{l}M \text { - Exploitations-Leistung der Hauptmaschine, } \\
Q_{t} \text { - Exploit.-Leistung der Hilfsmaschine, } \\
l_{s} \text { - Anzahl der Hilfsmaschinen } \\
n_{p}-\text { Verhältnis der Real-Leistung der Hauptmaschine zu ihrer Exploit.-Leistung während der Arbeits- } \\
\text { schichte (Ausnutzungsgrad der Maschine), } \\
n_{p}-\text { Ausnutzungsgrad der Hilfsmaschinen, } \\
a_{1}, a_{2}-\min . / \max \text {-Wert des } n_{s} \text {-Koeffizientes, } \\
b_{1}, b_{2}-\min \text {./max.-Wert des } n_{p} \text {-Koeffizientes }\end{array}$} \\
\hline
\end{tabular}




\subsection{Fall 1}

Der Verlauf der maximierten Verlustfunktionen gibt keine eindeutige Antwort, wenn es um die optimale Zahl $l_{s}$ geht. Es existiert kein Schnittpunkt dieser Funktionen, wie er in Abb. 6 oder in Tabelle 1 dargestellt wurde. Es besteht, daher die Notwendigkeit einer Verbindung der Auswahl einer optimalen $l_{s}$ mit Analyse mehrerer Kriterien.

Das Problem beschränkt sich auf ein strategisches Zwei-Personen-Nullsummenspiel, in dem der Zustand der Natur durch die Kriterirenfunktion widergespiegelt wird: Kranleistung, der benötigte Arbeitsaufand aller ausgeübten Tätigkeiten und Operationen, die Arbeitskosten des ganzen Teams. Da aber die Werteskala und die Maßeinheiten der einzelnen $f_{k}$ sehr verschieden sind, werden sie dimensionslose Werte überführt:

$$
w(m)=\frac{f_{k}-f_{k}(m)}{f_{k}^{o}}
$$

mit $f_{k}^{o}$ - Extremwert der Funktion [4].

Die Ergebnisse, für das Laplace- und das Wald-Kriterium, wurden in der Abb. 5 dargestellt.

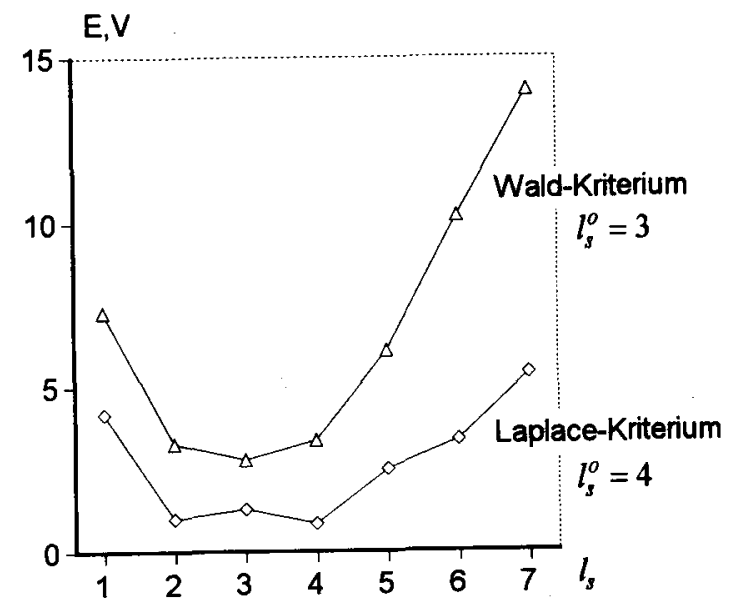

Abb. 5

Das Laplace-Kriterium, das von einem gesamten "Verlust" ausgeht ([5], [8]), weist auf ein 4 Personen starkes Montageteam hin. Die optimale Lösung für das Wald-Kriterium basiert auf dem für dieses Kriterium typischen Optimismus. Im Ergebnis der mehrkriteriellen Entscheidung auf der Basis des Laplace-Kriteriums soll berücksichtigt werden, daB die Umwandlungsregel der Ausgangsmatrix "Auszahlungen" (trotz der ursprünglichen Voraussetzungen) einen Einfluß der einzelnen Kriterien auf 
die das Endergebnis betreffende Abhängigkeiten ausüben kann. Zum Gegenstand der Analyse gehört also auch das Problem der Bestimmung der Zahl der in Beracht gezogenen Kriterien, und besonders dort, wo eine eindeutige Grenze nicht auftritt.

\subsection{Fall 2}

In Übereinstimmung mit der Abb. 4a und den durchgeführten Untersuchungen [9] ist

$$
\begin{aligned}
& T_{k r}^{\prime \prime}=0,091[\mathrm{~h}] \text { bei } n_{p} \in(0,73 ; 0,84), \\
& T_{b r}^{\prime \prime}\left(l_{s}\right)=0,168 \div 0,68[\mathrm{~h}] \text { fur } l_{s}=1, \ldots, 4 \text { bei } n_{s} \in(0,44 ; 2,87) .
\end{aligned}
$$

Die Maximalwerte der Verlustfunktion in Bezug auf die Montage einer Tunnelformvorrichtung sind für diesen Fall sehr leicht zu bestimmen und graphisch darzustellen (Tabelle 1). Der Schnittpunkt der Funktionen erscheint für $l_{s}^{o} \approx 4$

Die Empfindlichkeitsanalyse für die Optimallösung wurde in Bezug auf das Verhăltnis der Kranarbeitskosten zu den Kosten eines Angestellten durchgeführt. Die Ergebnisse für das höchste Harmonisienungsniveau für ein $l_{s}$ Personen starkes Team in Abhăngigkeit von dem genannten Kostenverhältnis, sind in Abb. 6 dargestellt.

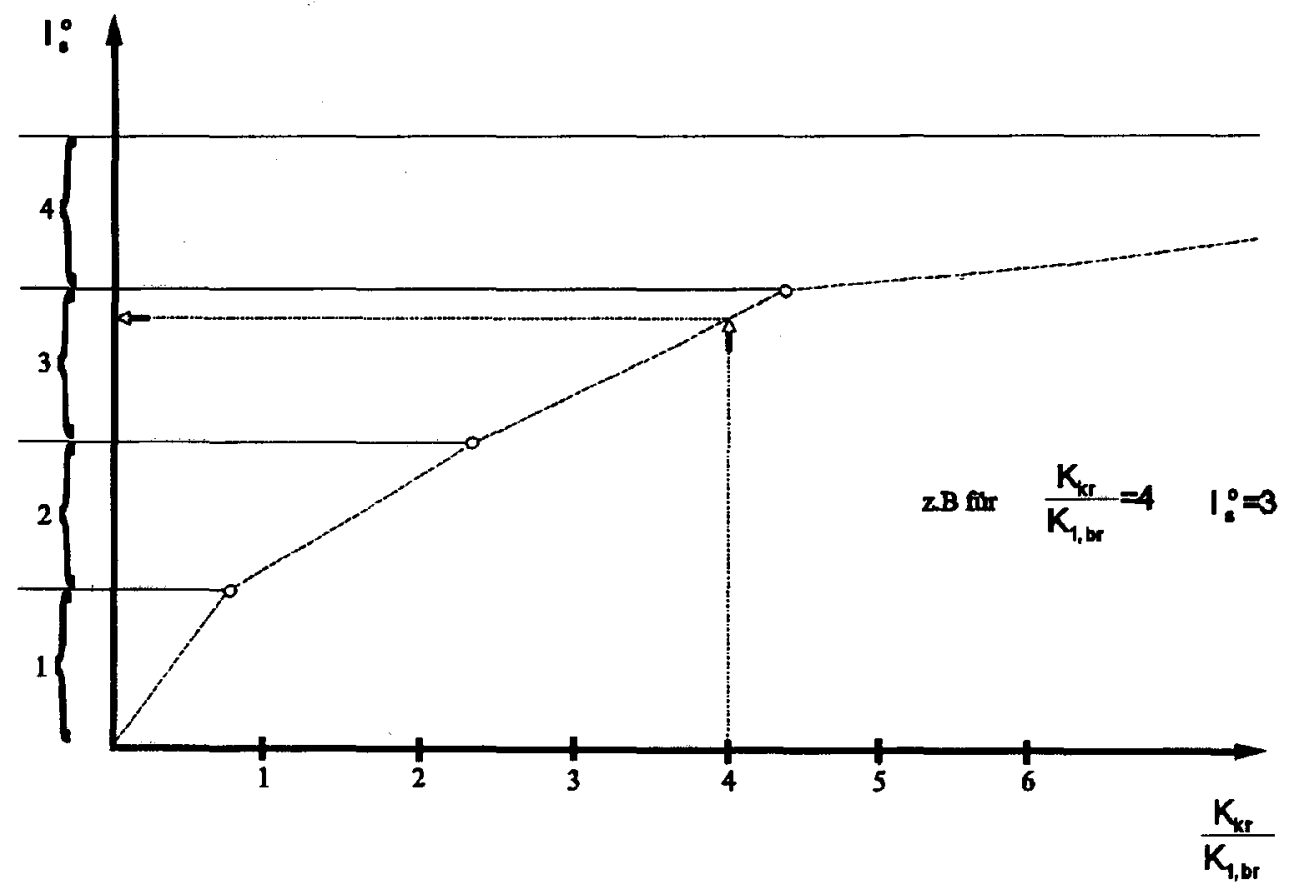

Abb. 6

Es besteht auch die Möglichkeit, die Analyse des Harmonisierungsniveaus nicht nur für die erschütterte Gleichgewichtserscheinung, sondern auch für das vollständige Problem durchzuführen. Wir werden also 
nicht nur, wie bisher, eine reine Strategie suchen, die uns eine eindeutige Zahlenstärke $l_{s}$ ausweist sondern interessieren uns auch für eine optimale Mischstrategie. Praktisch bedeutet dies, daß ein Team mit einer sich verändernden Zahlenstärke eingesetzt werden kann. Die Analysenergebnisse sind überraschend [9].

Das höchste Harmonisierungsniveau für den Fall 2 erhält man z.B. durch die Kombination eines 4Personen starken Teams (Häufigkeit $86 \%$ ) und eines 2-Personen-Teams (Häufigkeit $14 \%$ ). Die Überraschung geht vor allem aus einem großen, ungünstigen Wert der Verlustfunkion für ein 2Personen-Team hervor, daß alle Formvorrichtungen montiert, im Vergleich zu dem Wert der Verlustfunkion für ein 3-Personen starkes Team (das sich trotz des niedrigen Wertes der Verlustfunkion nicht in der optimalen Lossung befand). Aus dem oben genannten geht also hervor, daß ein a'priori Ausschließen von manchen Mischstrategien nur auf Grund des Verlaufes der Verlustfunkion für reine Strategien unbegründet ist.

\section{Literatur}

1. Höher G.: Ein Beitrag zur Verbesserung der Vorbereitung der Rekonstruktion von Wohngebä uden, Dissertation A, TH Leipzig 1982.

2. Kapliñski O. Meszek W.: Die Harmonisierung der Bauprozesse unter den Unbestimmtheitsbedingungen. Berichte vom VII MKÖ, Halle 1982, Band 2b S 59-61

3. Kapliñski O., Meszek W.: Catastrophic and strategic aspects of building processes balancing. Journal of Construction Management and Economics, 1992, Vol.10, pp. 81-88.

4. Kapliniski O., Hajdasz M., Meszek W.: Dobór brygad roboczych w warunkach nieokreslonosci. Berichte von XXIX. Konferenz KILiW PAN und KN PZITB, Krynica 1983, Band 3, S. 219 224.

5. Meszek W.: Harmonizacja procesow budowlanych w wanunkach nieokreslonosci. Dissertation A, Technische Universităt Poznañ, 1989.

6. Owen G.: Game theory. W.B. Saunders Company, Philadelphia, London, Toronto, 1968.

7. Peldschus F., Kapliñski O., Meszek W.: Zastosowanie teorii gier w przygotowaniu produkcji budowlanej. Wissenschaftliche Berichte Technische Universität Poznañ (Zesz. Nauk. Polit. Pozn., seria Budown. Ladowe), Nr. 34, 1990, S. 181-190.

8. Peldschus F.: Zur Anwendung der Theorie der Spiele für Aufgaben der Bautechnologie, Diss. B, Technische Hochschule Leipzig, 1986.

9. Rapport Nr. 64-061/81: Projektowanie ciagow technologicznych $w$ budownictwie $z$ uwzglednieniem badan niezawodnosci $i$ analizy zjawisk rownowagi. Teil III, Technische Universităt Poznañ, 1983. 
10. Selten R. (Ed.): Game equilibrium models, Vol. II. Methodes, Morals, and Markets. SpringerVerlag, Berlin-Heidelberg 1991.

11. Zavadskas E., Peldschus F., Kaklauskas A.: Multiple criteria evaluation of projects in construction. TECHNIKA, Vilnius 1994.

12. Zavadskas E.K.-K.: Sistemotechnitcheskaya ocenka technologitcheskich reshenii stroitelnogo proizvodstva, Strojizdat, Leningrad 1991.

13. Zavadskas E., Kaklauskas A., Bejder E.: Multiple criteria analysis of projects, Aalborg Universitetscenter, Aalborg, 1992.

\section{EINE ANALYSE VON ZYKLISCHEN SYSTEMEN UNTER UNBESTIMMTHEITSBEDINGUNGEN}

\section{O. Kapliñski}

Sum mary

This paper presents the problem of balancing specific construction processes with cyclic configuration. The processes of assembly of forming equipment in a monolithic housing building are an example of the consideration. The rules decomposition and synthesis at the production system has been used. Three examples of the loss functions are given. The results of analysis have been compared by means of Laplace's and Wald's criterions.

Der Dank

Dieser Aufsatz wurde teilweise mit einem Grant aus der Technischen

Universität Poznan No. DS: 11-023/95 unterstutzt. 